\title{
Diclofenac Sodium
}

National Cancer Institute

\section{Source}

National Cancer Institute. Diclofenac Sodium. NCI Thesaurus. Code C47984.

The sodium salt form of diclofenac, a benzene acetic acid derivate and nonsteroidal antiinflammatory drug (NSAID) with analgesic, antipyretic and anti-inflammatory activity. Diclofenac sodium is a non-selective reversible and competitive inhibitor of cyclooxygenase (COX), subsequently blocking the conversion of arachidonic acid into prostaglandin precursors. This leads to an inhibition of the formation of prostaglandins that are involved in pain, inflammation and fever. 